\begin{tabular}{|l|l|}
\hline Postprint Version & 1.0 \\
\hline Journal website & $\underline{\text { http://www.sciencedirect.com }}$ \\
Pubmed link & $\begin{array}{l}\text { http://www.ncbi.nlm.nih.gov/entrez/query.fcgi?cmd=Retrieve\&db=pubmed\&dop } \\
\text { t=Abstract\&list uids=15163486\&query hl=75\&itool=pubmed docsum }\end{array}$ \\
\hline DOI & $\begin{array}{l}10.1016 / j . v i r u s r e s .2004 .02 .010 \\
\end{array}$
\end{tabular}

\title{
Are influenza surveillance data useful for mapping presentations?
}

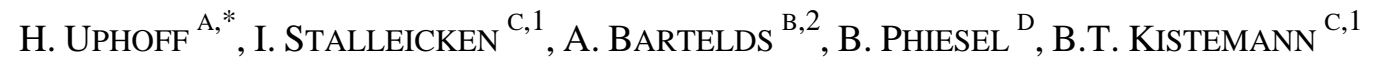

aAGI, Deutsches Grünes Kreuz, Schuhmarkt 4, 35037 Marburg, Germany

b Netherlands Institute for Health Services Research (NIVEL), PO Box 1568, 3500 BN Utrecht, The Netherlands

c Institute for Hygiene and Public Health, University of Bonn, Sigmund-Freud-Str. 25, 53105 Bonn, Germany

d Department of Geography, University of Bonn, Meckenheimer Allee 166, 53115 Bonn, Germany

* Corresponding author. Tel.: +49-6421-293142; fax: +49-6421-25730. E-mail addresses:

helmut.uphoff@kilian.de (H. Uphoff), ina.stalleicken@meb.uni-bonn.de (I. Stalleicken),

a.bartelds@nivel.nl (A. Bartelds), thomas.kistemann@ukb.uni-bonn.de (B.T. Kistemann). 1 Fax: +49228-2876763. 2 Tel.: +31-30-2729-700; fax: +31-30-2729-729.

\begin{abstract}
Geographical information system (GIS) based on mappings of influenza data are rare (http://www.b3e.jussieu.fr.80/sentiweb/fr) and influenza data are commonly aggregated for rather large areas (http://www.eiss.org, http://oms2b3e.jussieu.fr/FluNet). The most limiting factors for the use of morbidity-data from practices in GIS-based mappings are differences which are not related to morbidity. These differences may be due to consultation behaviour, interpretation of the case definition, age distribution of patients and other reasons. In order to reduce the impact of these non-morbidity related differences on the interpretation, the data of many practices are usually pooled and consequently rather large areas are presented. Extracting and harmonising the signals for increased morbidity from practices is a presupposition for mapping with a sufficient geographical resolution. The possibility to harmonise by reducing those confounding differences on a practice level is investigated.

Different harmonisation methods were applied to data from Germany where acute respiratory infections (ARI) per consultations are registered and from The Netherlands were influenza like illnesses (ILI) per population are registered. The harmonisation of the indices between countries was achieved by scaling them in relation to the level of the index representative for the peak activity during a usual influenza epidemic. The Kriging method is applied as a means of spatial prediction for the influenza data. The preliminary results are discussed with respect to resulting mappings.
\end{abstract}

\section{INTRODUCTION}

Surveillance of the morbidity due to influenza relies usually on syndromic data from primary care facilities, combined with virological data from the same source. Virological data are commonly interpreted in a more qualitative way — is there influenza circulating? and which viruses are active?while the syndromic data are used for quantitative analyses. The interpretation of the syndromic data 
in general is based on the change in morbidity - the influenza-attributable excess morbidity-in relation to the excess seen in previous seasons. This basic concept is used in The Netherlands and in Germany as well as in most EISS member networks and other countries (Fleming et al., 1999; Manuguerra and Mosnier, 2000).

The magnitude of the influenza-attributable morbidity as an indicator for the influenza activity can be well assessed with pooled data from many practices in relation to comparable data of previous seasons. Regardless of the different numerators and denominators used and the different national health care systems influenza activity can be detected by the surveillance systems and the influenza activity can be assessed by appropriate methods (Manuguerra et al., 2001; Snacken et al., 1995).

Nevertheless within each sentinel network, data from individual practices show considerable differences and variation which can be attributed to several factors (Table 1).

\section{[ TABLE 1 ]}

Some factors such as consultation patterns and morbidity of the different age groups, interpretation of the case definitions, etc. induce rather continuous or long-term differences while others, e.g. opening hours, holidays, closed neighbouring practices, etc. cause short-term changes (Uphoff, 1998a).

In particular, continuous differences between practices limit the direct use of the data for mapping because they constantly interfere with the morbidity related differences. This interference can be reduced by excessive spatial smoothing which reduces the sensitivity for geographical differences or by other harmonisation methods. In France, where mapping of influenza surveillance data has been done for many years, the so-called over-reporting factor is used to reduce those differences not related to morbidity (Schlaud, 1999; Carrat and Valeron, 1992).

Harmonisation of data within the sentinel networks by uniformly scaling the practice data may allow a useful compromise between requirement for smoothing and sensitivity for geographical patterns. The impact and kind of the confounding factors may differ between the sentinels depending on its set up and design, the health care system and other presuppositions. As a consequence the suitability of the data for mapping may differ between sentinels as well as the requirements and possibilities for harmonisation. With the two examples of the networks in The Netherlands and Germany preliminary harmonisation procedures are explored.

Nation-wide mapping of morbidity data is often disturbed by the so-called border effect. Due to a lack of comparably scaled data across the border the geographical smoothing is distorted in border areas. This problem increases with a low density of measuring points and is a particular problem in small countries. For influenza surveillance the density of practices is rather low and the border effect clearly is a disadvantage for a nation-based Europe-wide mapping. While national borders do not function as morbidity barriers for influenza a pan-national mapping of the data appears desirable. All countries interpret their indicators for influenza activity in relation to previous experiences and give interpretations like usual influenza activity, more than usual, etc. This congruence might provide the platform for a uniform scaling of the indicators used. Such a uniform scaling is a presupposition for a mapping which overlaps borders.

\section{MATERIAL}

\subsection{Germany}

In Germany acute respiratory infections (ARI) are registered in more than 500 primary care practices (1\% of all practices). Cases of acute pharyngitis, bronchitis and pneumonia with and without fever are recorded in five age groups on a weekly basis. This is definition that includes more than just influenza typical syndromes and is closer to acute respiratory infections. Records of holiday weeks with no consultations are not considered, while records with reduced opening time in a week (opened from 1 day a week up to the whole week open) are included in the processing. The morbidity data cannot directly be linked to population on a practice level (free choice of the practice contacted) and hence the number of consultations is used as a denominator giving the indicator ARI per 100 consultations. The surveillance includes three groups of specialised practices in primary care (generalists, internists and paediatricians) with a slight over representation of paediatricians (19\%). The network has a 10 
15\% fluctuation of practices annually. The data from the seasons 1994/1995 to 1999/ 2000 were used for the development of the harmonisation methods.

Particularly in the heterogeneous network of Germany continuous differences between practices are large and temporal variation has also to be considered (Uphoff, 1998a).

In Fig. 1 the practices are split up into four groups as regards the level of ARI per 100 consultations in the pre-epidemic period. Each group consists of between 60 and 150 practices and the practices in each group have a rather random spatial distribution. The differences in the levels of this indicator are continuous over the year (and years/not shown) while the increase in morbidity when influenza circulated is obvious in each group. Paediatric practices are accumulated in the group with a high level of ARI per 100 consultations as respiratory infections are very common in this age group.

\section{[ FIGURE 1 ]}

\subsection{The Netherlands}

In The Netherlands influenza like illnesses (ILI) (acute onset, temperature above $38{ }^{\circ} \mathrm{C}$, and one or more of the following symptoms: cough, coryza, sore throat, frontal headache, retrosternal pain, myalgia) are registered in about 53 practices. The network is very stable. The ILI cases registered can be directly linked to the population each practice cares for and the network covers $1 \%$ of the population. The surveillance is solely based on generalists and the ILI per 100,000 population is used as indicator for the influenza-related morbidity. Only weeks with more than three days opening time are considered, which reduces the number of records considered during holiday seasons (like Christmas and New Year) but also reduces temporal distortions of the indicator in those periods (Bartelds, 1993). Data from the seasons 1989/1990 to 1999/2000 were included for the set up and testing of the harmonisation method used for The Netherlands.

The consideration of the population covered by each practice as denominator allows a better standardisation of the indicator for influenza activity. Continuous differences between practices are smaller than in the heterogeneous sentinels of Germany but are still obvious when the practices are split up into three groups according to the median of the peak levels (three consecutive peak weeks per season over all seasons available for the practice with a minimum of five recent seasons available (Figs. 2 and 3)). The groups consist of 12 practices each and the group with consistently low values accumulates in the middle and north parts of the country. The practices with median values are in the middle and the south while the practices with high values are rather randomly distributed geographically.

\section{[ FIGURES 2-3 ]}

Due to the different methods and presuppositions the crude indicators used by the two surveillance systems differ considerably (Fig. 4).

\section{[ FIGURE 4 ]}

\section{METHODS}

\subsection{Harmonisation}

\subsubsection{Germany}

For Germany an index (practice-index) has been used for regional presentation of weekly data (Uphoff, 1998b). This practice-index could significantly reduce the differences between practices in the level (compare Fig. 1) of the parameter and could reduce temporal distortion due to reduced opening times of practices. The index was calculated for each practice using the change of two indicators (ARI per 100 consultations and ARI per practice) in relation to a level of those indicators 
representing the background or expected level for each practice. The background or expected level was estimated for each practice from the pre-epidemic phase as a mean over all weekly records available up to the start of influenza activity:

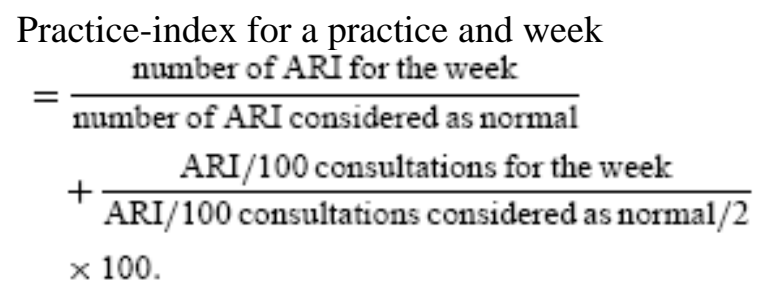

This is the change of the morbidity indicator in percent of the normal level for each practice. Due to the large differences of the background levels the relative increase of the index during influenza activity differed considerably with the magnitude of the background level. A second disadvantage of this practice-index resulted from the fixed value for the background level (mean of pre-epidemic weeks) whereas in reality the expected background changes by week followed a characteristic curve over the year. This disadvantage could be reduced by the consideration of the usual yearly course of the practice-index as base level.

The different increases of the practice-index-because of the relation to the widely differing background level—-was corrected by an empirically estimated factor for each group in a preliminary attempt:

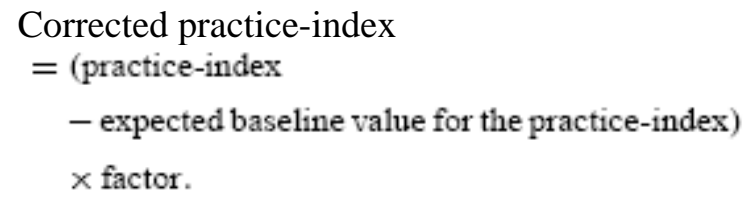

This corrected practice-index has been used for mappings of the weekly ARI morbidity levels in Germany (http://influenza.rki.de/agi) (Uhoff and Phiesel, 2002).

\subsubsection{The Netherlands}

In order to reduce continuous differences between the practices the weekly ILI per 100,000 for each practice was set in relation to the usual peak level of each practice. This usual peak level is represented by the mean of three consecutive peak values of each of the seasons available for the practice from 1989 to 2000. Practices with less than five seasons available were excluded:

$$
\begin{aligned}
& \text { Index for each practice and week } \\
& =\frac{\text { ILI per } 100,000 \text { for the week }}{\text { usual peak level }} \times 100
\end{aligned}
$$

\subsubsection{Scaling of the two national indexes}

The uniform scaling for both indexes was achieved by relating the weekly index value for each practice to an index value observed during the peak of influenza epidemics considered as usual. By this operation the turn of the scale typical for each system was taken into account and a uniform scaling with 0 (no signal for excess morbidity) and 100 (excess morbidity typical for a usual influenza epidemic) was achieved (Uphoff, 1998c).

The case definition in the system of The Netherlands is rather specific and the "background" morbidity is low and often zero on a practice level. Negative index values—signalling less than usual morbidity-are not sufficiently indicated. The index for The Netherlands represents the percentage of increase of the ILI consultation incidence in relation to an average peak level for each practice, and therefore signals only excess morbidity and cannot be negative. In Germany the "background" morbidity is large and the index (in relation to the "background") can become negative indicating less than usual morbidity. To exclude interference and distortions with negative index values for Germany 
at the borders to The Netherlands where negative values cannot occur, the negative values for Germany were set to 0 : no signal for excess morbidity. This provides a better congruence with the data from The Netherlands.

\subsubsection{Geographical analysis}

For the following spatial analysis all practices with less than seven reports for the year were excluded. The practices were located by the five digit postal-code for Germany (Phiesel, 2001) and the four digit postal-code for The Netherlands. By this 12,264 areas of comparable dimensions were considered for Germany and 3994 for The Netherlands. This did not allow the exact location of the practice as the centre of the area coded was used as location (Fig. 5). From the 12,264 postal-code areas in Germany practices could be assigned 3.7\% of the areas with one practice in 437 areas, two practices in 22 areas and three practices in two areas. In The Netherlands practices could be assigned to 46 areas with two practices in three areas (1.1\% of the areas).

\section{[ FIGURE 5 ]}

This allowed the consideration of at least 12 values (practices) for the interpolation. Ordinary Kriging was used for the smoothing of the weekly data, assuming that the data set has a stationary variance but also a non-stationary mean value within the search radius. Such a kriged estimate is a weighted linear combination of the known sample values around the point to be estimated. The range, representing the distance at which there is no longer a correlation between the points, was $40-50 \mathrm{~km}$. This allowed the consideration of at least 12 values (practices) for the interpolation.

\section{RESULTS}

\subsection{Germany}

The corrected practice-index was considered acceptable as harmonisation between the groups of practices which is plotted for the 1999/2000 season in Germany (Fig. 6).

\section{[ FIGURE 6 ]}

\subsection{The Netherlands}

For The Netherlands a datum point as reference for the index was estimated from the three peak values for each practice of the seasons available (1989-2000). This reference for each practice was set at $100 \%$ and the relation of the weekly value as percent of the reference was plotted (Fig. 7). This index appeared acceptably uniform for the data from The Netherlands.

\section{[ FIGURE 7 ]}

The harmonised index values for both networks reflect the course and intensity of influenza activity as interpreted by the classical methods. The index indicates a similar intensity of the 1999/2000 season in both countries which may be characterised as usual to pronounced activity during the peak (Fig. 8). The beginning of the influenza epidemic in Germany is one week later than in The Netherlands and the peak in Germany is delayed for one or two weeks. The end of the influenza epidemic is 1-2 weeks later than in The Netherlands. This may result from the larger geographical dimensions of Germany and is congruent to the observations in the neighbouring countries (Manuguerra and Mosnier, 2000). In the season 1999/2000 the activity started in the west (France) and moved to the east affecting eastern regions after a delay.

\section{[ FIGURE 8 ]}


After the harmonisation the weekly index curves (Fig. 7) as well as the variation coefficients of the index values (Fig. 9) over all practices of The Netherlands and Germany are fairly similar.

\section{[ FIGURE 9 ]}

\subsection{Spatial analysis}

The assignment of the values to the centre of the artificial postal-code areas can cause a misplacement of up to $15 \mathrm{~km}$ in a few areas but in general the shift is much smaller.

The weekly variogrammes and mappings indicate low spatial variation during the pre- and postepidemic phases.

The index is in a normal range in the majority of practices with some irregularly distributed practices with high and low values shifting during the weeks.

When the influenza activity increases, it is especially the number of practices with elevated index values which rises and areas with elevated index values that can be recognised. The development of these foci are congruent to the classical regional analysis. Besides a tendency of a geographical spread additionally clusters and practices with high indicator values arise spontaneously outside the suggested diffusion areas. At the peak of the influenza activity in weeks 1-3 (NL) and 3-5 (D) the morbidity is elevated in the entire area but to different levels. In both countries the northern districts seem less affected than the southern areas (Figs. 10 and 11). The spatial differences decline with the influenza activity and are minor in the post-epidemic period with a few condensed foci. In general a west to east movement can be recognised which is in line with the observations in the neighbouring countries There are no obvious shifts in the border area of the two countries.

\section{[ FIGURES 10-11 ]}

\section{DISCUSSION}

Interpretation of data from primary care practices are difficult and knowledge regarding the registration procedures in the practices is needed and empirical experience is helpful. Detail is lost due to the different aggregation processes which are usually necessary to reduce the bias. Neither the relation between the incidence of influenza in the population and the indicator used by a surveillance system nor the true incidence of the syndrome in the population covered by the practices is exactly known. Hence the interpretation of the aggregated data is commonly based on the relation to previous experience with the same system. Therefore a stable system and reporting is important and long-term changes particularly for the non-epidemic periods (baseline) are important and have to be considered. Long-term changes in the peak activity can only be recognised after many years due to high yearly variation and there are no clear evaluation procedures for that at the moment. Apparent changes can be considered for the mapping if necessary during the scaling process by changing the values for the usual activity.

Harmonising the data between practices and networks and applying spatial interpolations provides an additional perspective on the surveillance data.

For spatial analysis it is important to reduce the influence of confounding factors and the differences between single practices not due to the morbidity in the population covered. Harmonisation and reduction of those confounding influences is achieved by a consistent application of the principle to assess the influenza activity in relation to previous experiences on a practice level.

For the intra-system harmonisation the basic step is setting the indicator or indicators used in relation to a reference value. This step decreases the influence of factors which are presumably not related to morbidity differences between the practices. Continuous differences between practices or groups of practices which are consistent for many years are reduced which is stringent to the principle of interpretation in relation to previous experiences. In particular for the harmonisation within the systems it appears questionable if those continuous differences are morbidity related. (There are no indications found in the literature for local gaps in population immunity against influenza in European countries by sero-surveillance data.) 


\subsection{The Netherlands}

In The Netherlands the network and the data are more homogenous. A satisfactory harmonisation was achieved by a relation of the indicator to the average of the peak influenza activity on a practice level. Interestingly the groups compared did not give the impression of a spatially even distribution, but this has not been tested by cluster analyses or other methods as yet. In case there is a low influenza activity constantly reported from a certain region, a comparison of sero-surveillance data with a region reporting a high influenza activity may be helpful. On the other hand, these regional differences might be explained by discussion among the physicians regarding, e.g. the interpretation of the criteria during meetings, etc. or regional differences in consultation behaviour.

Nevertheless these differences were largely levelled out by the index due to the relation of the indicator for influenza activity to previous experience on a practice level.

\subsection{Germany}

In Germany the participating practices are more heterogeneous and the differences in the level of the indicator and the increase during phases of elevated influenza activity are large. As indicated by the analyses of the groups both differences could be well harmonised. Due to the consideration of weekly values with considerably reduced opening time distortions and differences particularly during Christmas and New Year remain and may not be morbidity related. Some methodological options have to be explored to further reduce these influences. The consideration of already corrected values for the expected curve of the weekly base values and/or the consideration of the correction factor before the expected base values are considered-may be an option. The differences in the Christmas holiday season may be partly due to the high proportion of paediatric practices in the groups with high "background values" and reduced morbidity particularly in the younger age groups due to school holidays or changes in consultation behaviour happening particularly in those age groups.

\subsection{International harmonisation}

The harmonisation between the systems based on the previous experience and the traditional considerations of the activity for each system is useful and takes traditional interpretation principles and assessments into account. The use of such assessment has an arbitrary momentum but the degree of "unsharpness" — or lack of precision—seems tolerable in relation to other influences. The reference data point chosen-normal influenza epidemics—is in a range that is frequently reached and less prone to distortions that may occur during extreme situations like during severe epidemics.

Ignoring the negative index values for Germany is justifiable because solely the signals for excess morbidity are picked up in The Netherlands and indeed are of great interest. This operation shifts the variation coefficient for both networks to the same level which may be seen as an additional indication for a good harmonisation.

A sufficient degree of harmonisation is also indicated by the index curves which well reflect the national assessments of the season (van den Brandhof et al., 2001; AGI, 2000). Sharp shifts at the border do neither occur during the pre-epidemic phase nor during the influenza epidemic which underlines the good international harmonisation. The results are congruent to conventional regional analysis as well as to the national assessments of the influenza activity in the two countries and the neighbouring countries (Manuguerra and Mosnier, 2000).

Crossing-border spatial analysis of harmonised index values seems possible and helpful for a detailed spatial analysis. System immanent differences between the systems like, e.g. a higher sensitivity and hence turn of the scale for ARI based sentinels for H1N1 or B activity (with a lower proportion of typical ILI) and vice versa are still present and have to be further explored and considered for interpretation.

\subsection{Spatial analysis}

The mistake in the precise location of the practices representing a relatively large area seems tolerable in relation to the range used. The exact area covered by a practice is not known and these mistakes of the area covered and the location of the practices are inevitable. The number of practices considered for the index value appointed to an area depends on the range used. Finding the best possible compromise between smoothing and discrimination of spatial difference (range), is related to an understanding of the degree of harmonisation and the geographic diffusion of influenza. Carrat and Valeron estimate the consideration of 12-20 values as acceptable for spatial interpolation [10]. Due to 
the relatively low density of measuring points the range was about $50 \mathrm{~km}$ providing the consideration of at least 12 practices for the interpolation. As no hypothesis regarding the geographical diffusion of influenza is present, a uniform range and distribution model used for all weeks appears sufficient in assessing the temporal and spatial changes. The absence of spatial differences is also useful information and the low spatial differences during the pre- and post-epidemic periods may suggest an acceptable harmonisation.

\subsection{Perspectives}

It has been demonstrated that mapping of influenza surveillance data across borders is possible. The option of further improving the harmonisation has to be explored and seems achievable particularly by improving the harmonisation- factor and reducing the distortions due to reduced opening times in the German system.

The application of the methods discussed and adaptation of the methods to other countries should also be tested. The comparability of the data from different countries is of particular interest and the pan- and international harmonisation options have to be reconsidered with more countries and seasons included.

The spatial analyses (possibly by using a set of ranges and models) should be applied to different observation periods to achieve a better understanding of these effects and to give a better idea of the diffusion of influenza. A better understanding of the diffusion of influenza might enable us to improve the spatial smoothing or interpolation procedures.

The usefulness of this additional perspective on influenza surveillance data and the quality and interpretation of the resulting maps have to be explored carefully.

\section{ACKNOWLEDGEMENTS}

We thank all the colleagues in the EISS group for the fruitful discussions and comments. We additionally thank all the physicians supplying the data without whom the national surveillance would not be possible.

\section{TABLES AND FIGURES}

\begin{tabular}{ll} 
Table 1 & \\
\hline Morbidity & $\begin{array}{l}\text { Changes in morbidity due to influenza or to other } \\
\text { agents }\end{array}$ \\
$\begin{array}{l}\text { Practice-type } \\
\text { Interaction }\end{array}$ & $\begin{array}{l}\text { Consultation behaviour of the population in relation } \\
\text { to age, culture, education, holidays, etc. }\end{array}$ \\
& $\begin{array}{l}\text { Organisational needs like an official confirmation of } \\
\text { illness for the employer, etc. }\end{array}$ \\
Selection & $\begin{array}{l}\text { Criteria given, interpretation of the criteria, etc. } \\
\text { Opening time, practitioner on duty, home visits, } \\
\text { closed neighbouring practices, locum, holidays, etc. }\end{array}$ \\
\hline
\end{tabular}


Uphoff, H., Stalleicken, I., Bartelds, A., Phiesel, B., Kistemann, B.T. Are influenza surveillance data useful for mapping presentations?

Virus Research: 103, 2004, nr. 1-2, p. 35-46
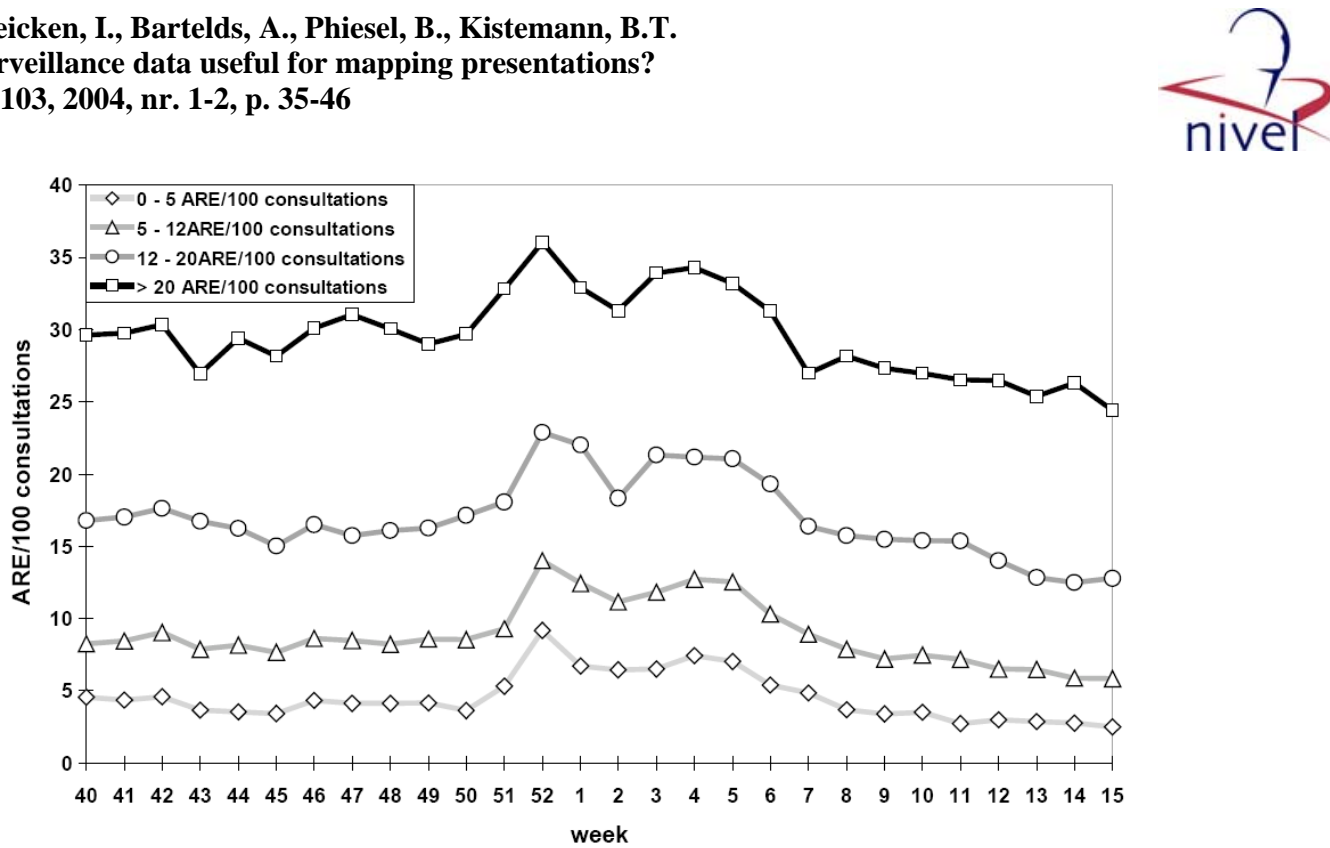

Fig. 1. ARI per 100 consultations in Germany for the season 1999/00 in four categories of practices, selected regarding the level of this parameter in the pre-epidemic period.

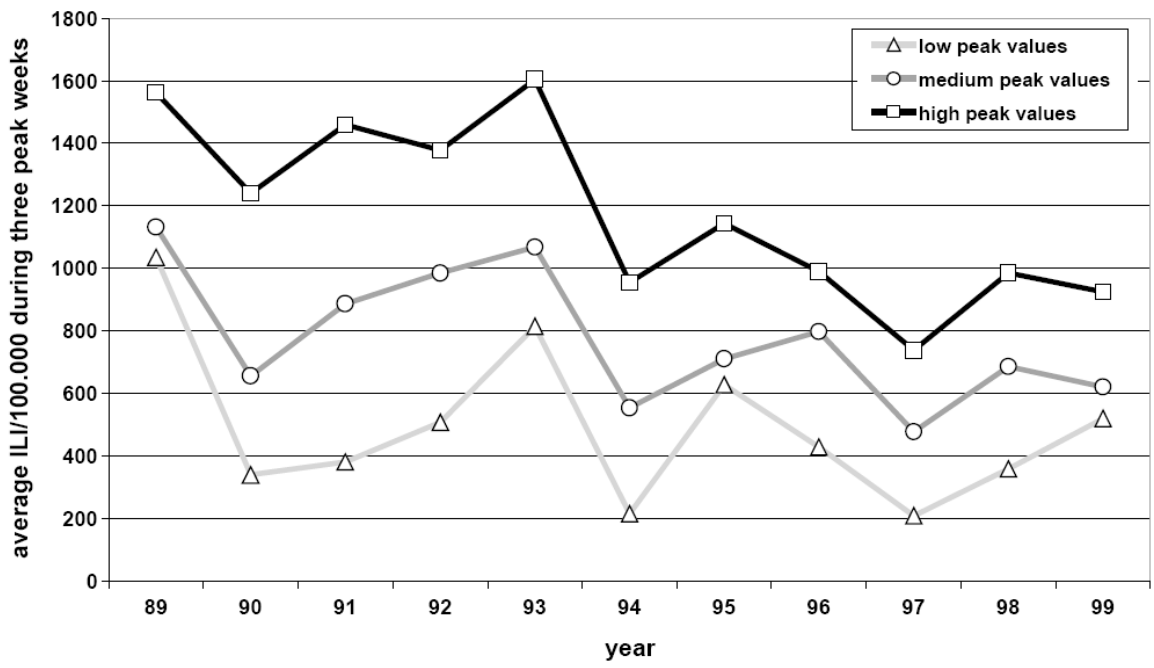

Fig. 2. Mean of three peak incidence weeks per practice and season plotted as average for three groups of 12 practices each-selected for high, medium and low median values over all seasons. Season 1989/1990 to 1999/2000 in The Netherlands.

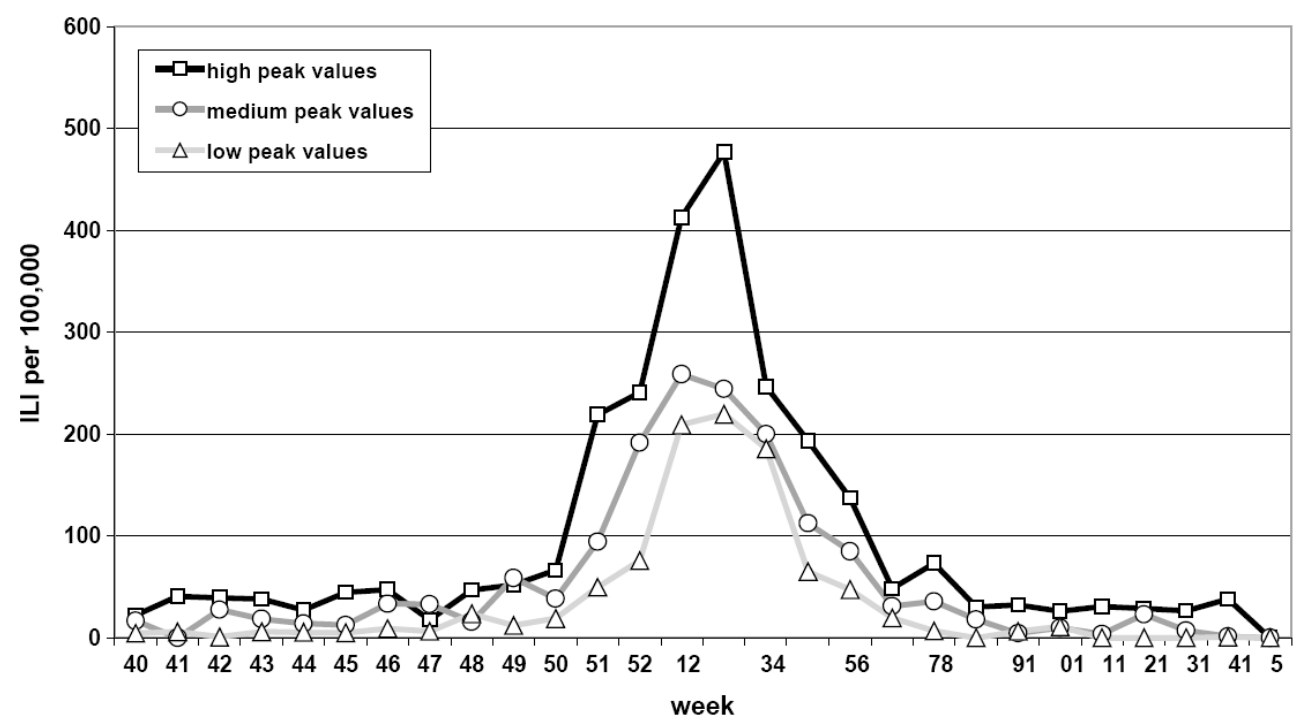

Fig. 3. ILI per 100,000 as mean value for each of the three groups of practices for the season 1999/2000 in The Netherlands. 
Uphoff, H., Stalleicken, I., Bartelds, A., Phiesel, B., Kistemann, B.T.

Are influenza surveillance data useful for mapping presentations?

Virus Research: 103, 2004, nr. 1-2, p. 35-46
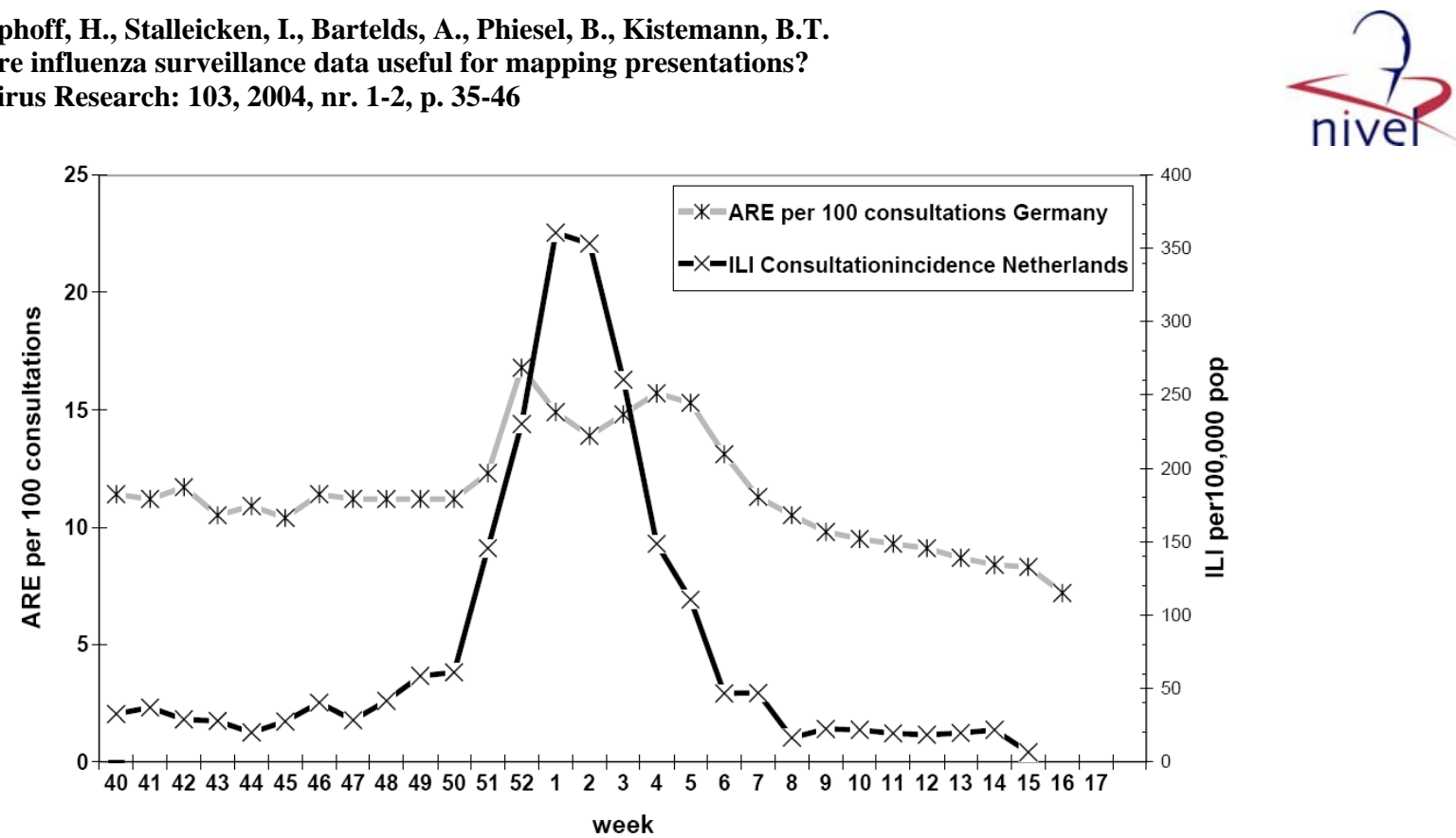

Fig. 4. Consultation incidence of ILI (The Netherlands) and ARI per 100 consultations (Germany) during the season 1999/2000

\section{Distribution of practices in 1998/99}

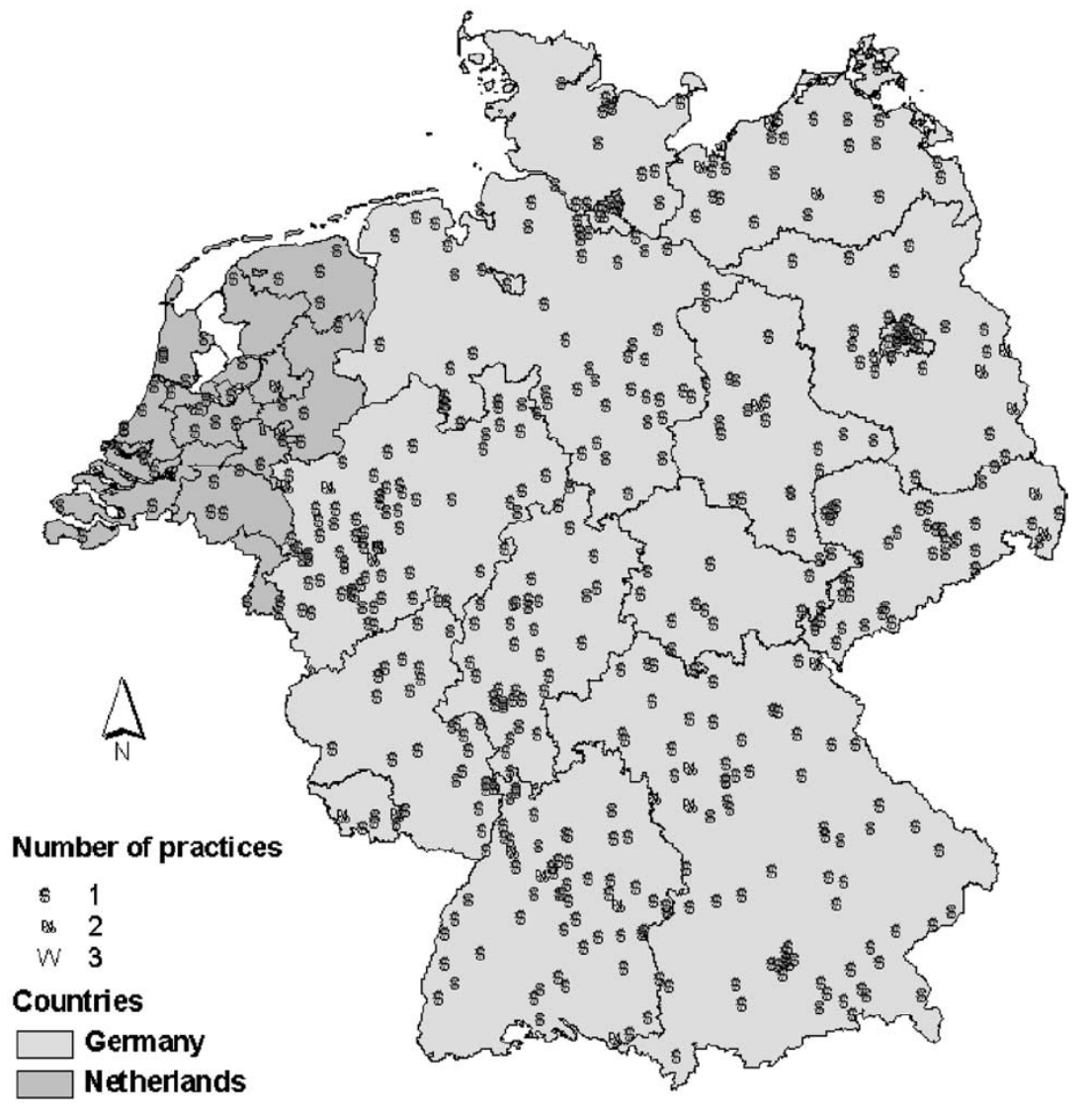

district boundaries

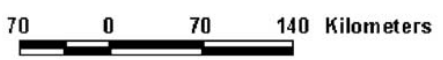

Data source: AG Influenza 1998/99

Cartography and design

In stitute for Hygiene and Public Health, University of Bonn 2002

Fig. 5. Corrected practice-index of the season 1999/2000 in Germany given in four categories of practices, selected regarding the level of ARI/100 consultations in the pre-epidemic period and levelled for usual influenza epidemic $=100 \%$. 
Uphoff, H., Stalleicken, I., Bartelds, A., Phiesel, B., Kistemann, B.T. Are influenza surveillance data useful for mapping presentations?

Virus Research: 103, 2004, nr. 1-2, p. 35-46
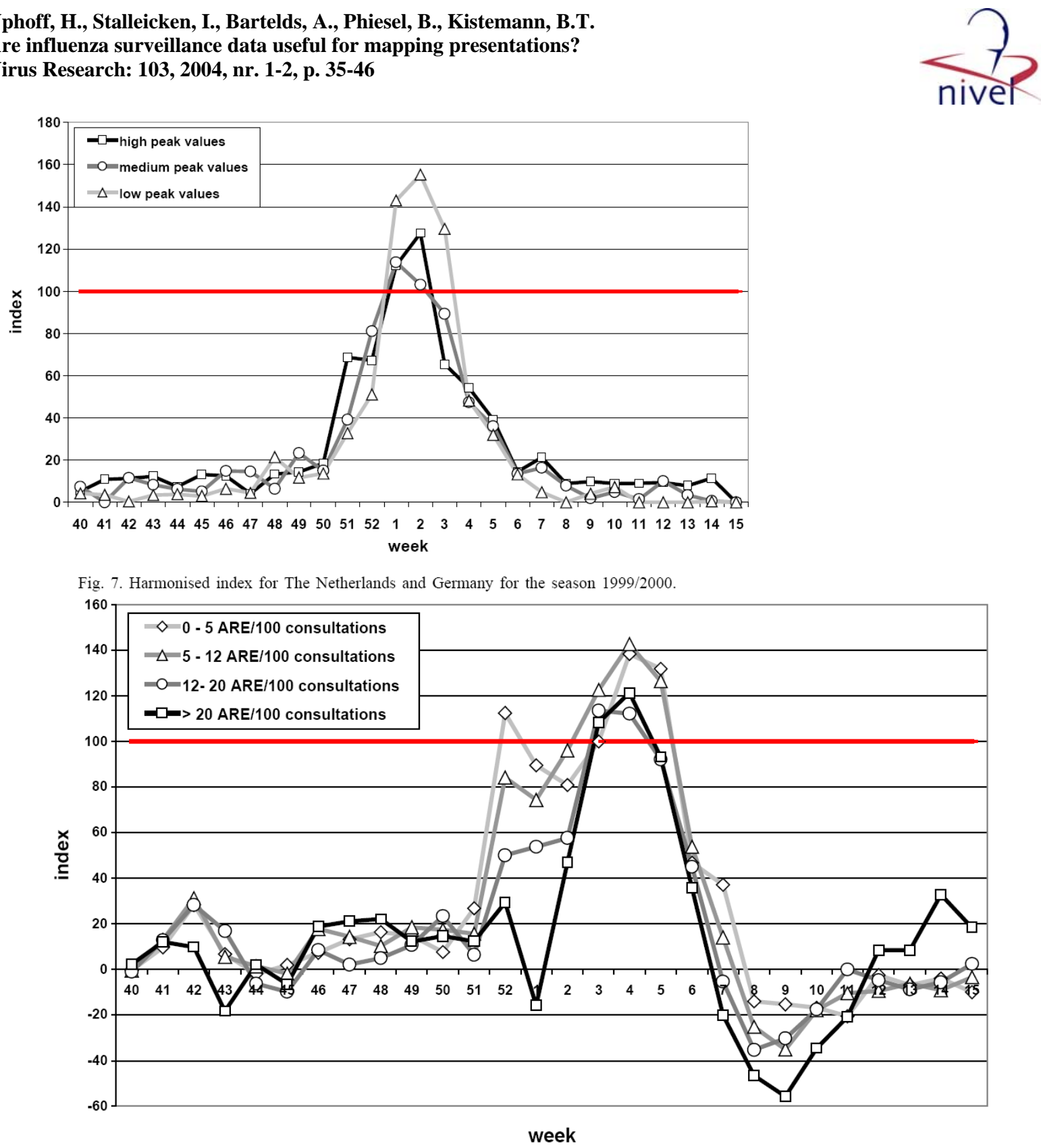

Fig. 6. Index for The Netherlands during the season 1999/2000 in three groups classed referring the median of the peak values in the seasons $1989-2000$, levelled for usual influenza epidemic $=100 \%$. 
Uphoff, H., Stalleicken, I., Bartelds, A., Phiesel, B., Kistemann, B.T.

Are influenza surveillance data useful for mapping presentations?

Virus Research: 103, 2004, nr. 1-2, p. 35-46
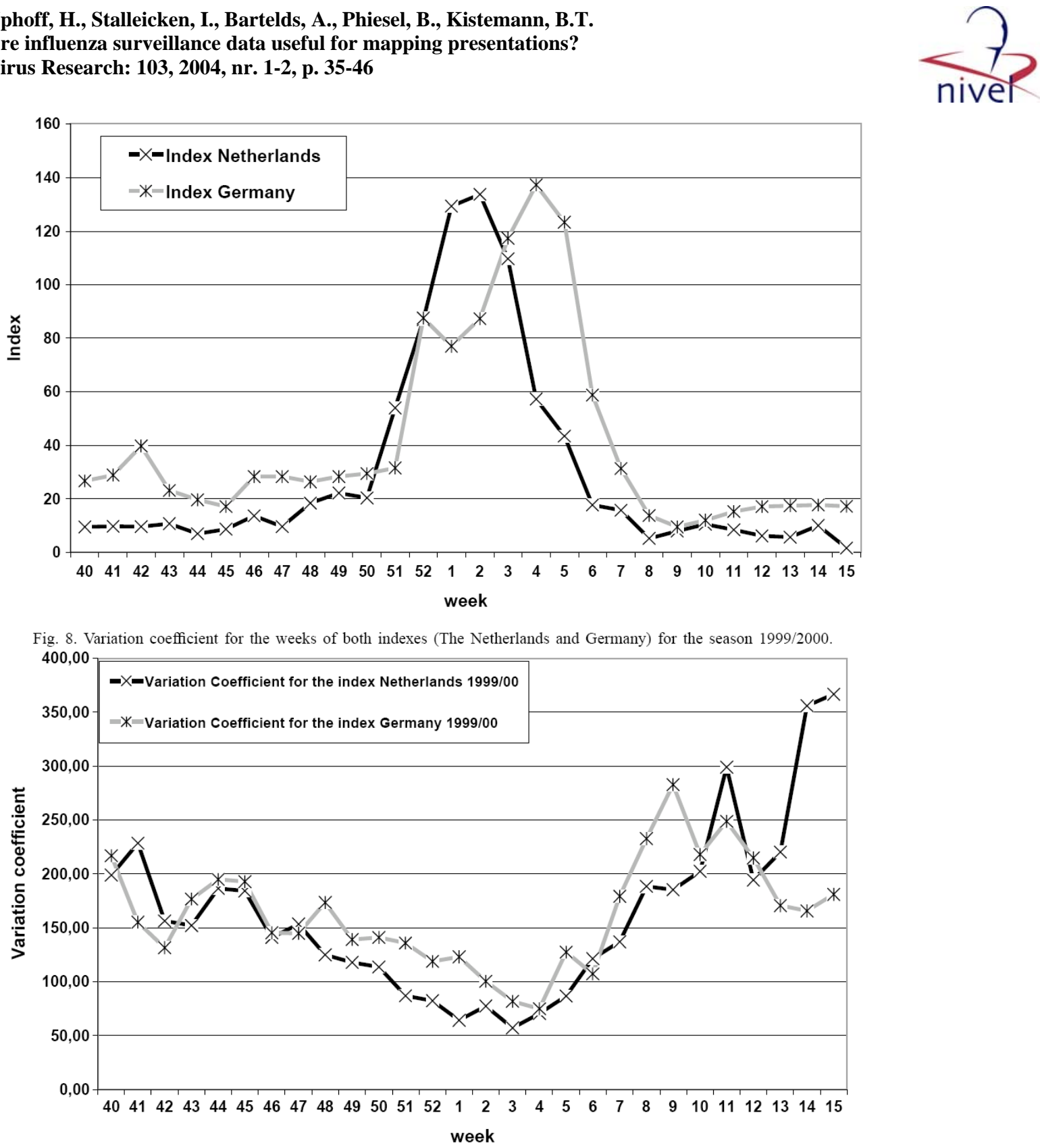

Fig. 9. Geographical distribution of the practices in the season 1999/2000. 
Uphoff, H., Stalleicken, I., Bartelds, A., Phiesel, B., Kistemann, B.T. Are influenza surveillance data useful for mapping presentations? Virus Research: 103, 2004, nr. 1-2, p. 35-46

\section{Spatial distribution of Influenza in 1999/2000} - Interpolation via kriging -

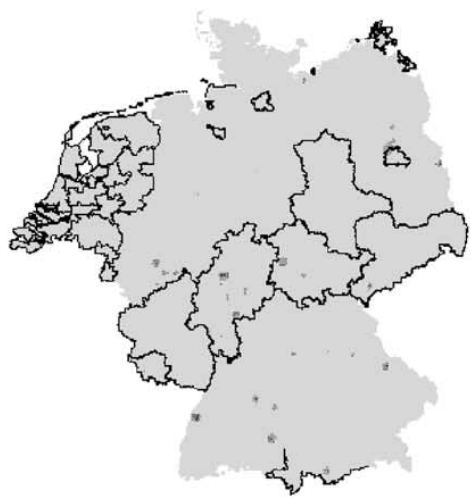

calend ar week no. 50

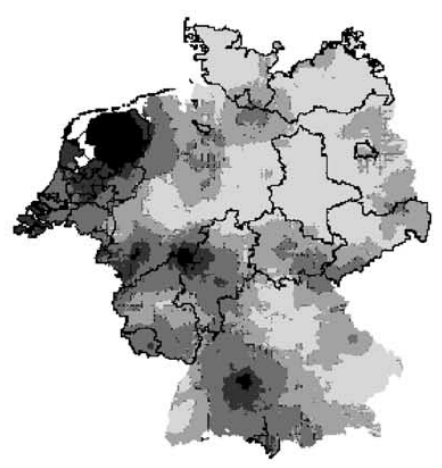

calend ar week no. 1

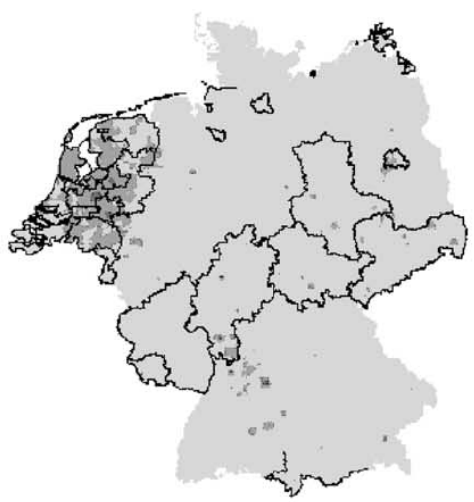

calendar week no. 51

$\triangle$

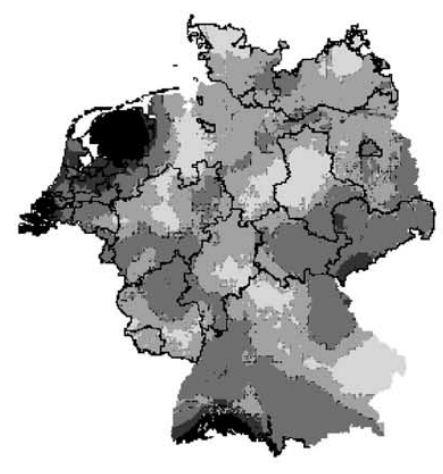

calendar week no. 2

Practice index (corrected II)

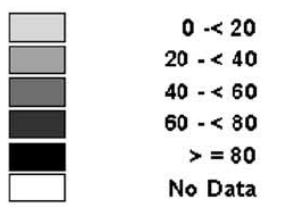

Data source:

AG Influenza 1999/ 2000

- district boundaries

Fig. 10. Mappings of the harmonised index values for the weeks 50 of the year 1999 to 2 of the year 2000 . 
Spatial distribution of Influenza in 1999/2000 - Interpolation via kriging -

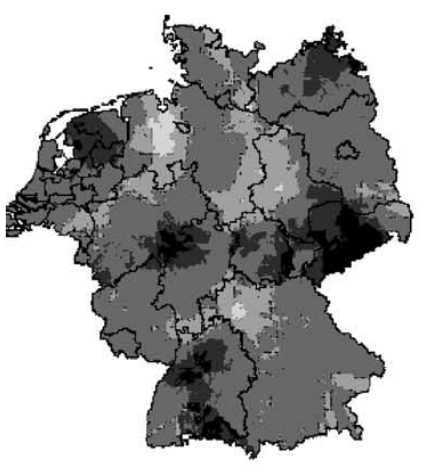

calend ar week no. 3

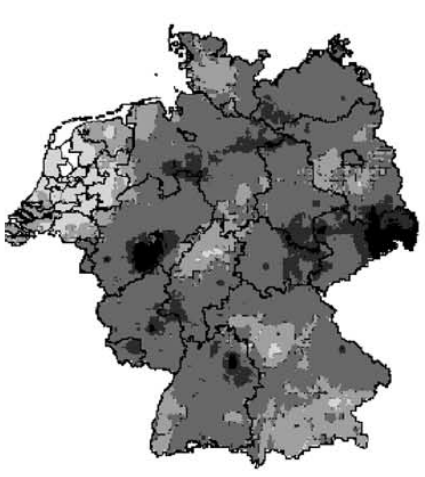

calend arw eek no. 5

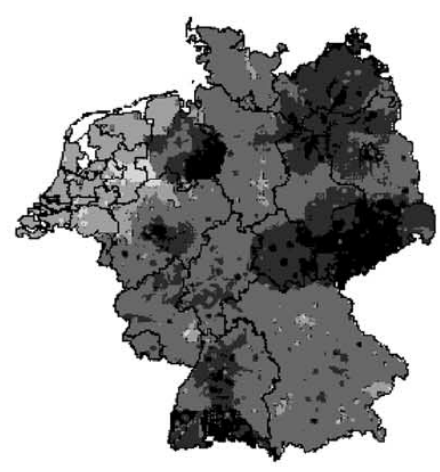

calendar week no. 4
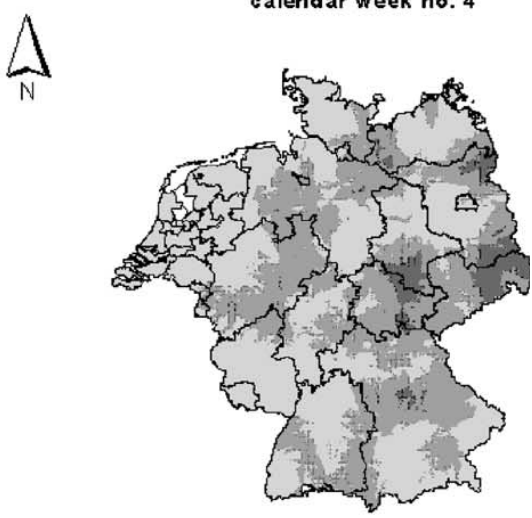

calendar week no. 6

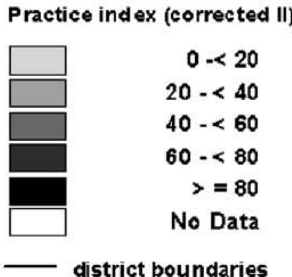

district boundaries

Data s ource:

AG Influenza 1999/2000

Cartography and design:
Institute for Hygiene and Public H ealth, University of Bonn 2002

Fig. 11. Mappings of the harmonised index values for the weeks 3-6 of the year 2000.

\section{REFERENCES}

1. AGI (Arbeitsgemeinschaft Influenza), 2000. Ergebnisse der Meldephase 1999/2000, Marburg.

2. Bartelds, A.I.M., 1993. Zur Validierung von Sentinel Daten. Das Gesundheitswesen 55, 3-7.

3. Carrat, F., Valeron, A.J., 1992. Epidemiologic mapping using the "Kriging" method: application to an influenza-like illness epidemic in France. Am. J. Epidemiol. 135, 1293-1300.

4. Fleming, D.M., Zambon, M., Bartelds, A.I.M., de Jong, J.C., 1999. The duration and magnitude of influenza epidemics: a study of surveillance data from sentinel general practices in England, Wales and The Netherlands. Eur. J. Epidemiol. 15, 467-473.

5. Manuguerra, J.-C., Mosnier, A. (on behalf of EISS), 2000. Surveillance of influenza in Europe from October 1999 to February 2000. Eurosurveillance 5, 63-68.

6. Manuguerra, C., Mosnier, A., Paget, W.J., 2001. Monitoring of influenza in the EISS European network member countries from October 2000 to April 2001. Eurosurveillance 6, 127-135.

7. Phiesel, B., GIS-gestützte Analyse der raum-zeitlichen Verbreitung von Influenza in Deutschland, 2001. Diplomarbeit an dem Geographischen Institut der Rheinischen FriedrichWilhelms-Universität Bonn.

8. Schlaud, M., 1999. Comparison and Harmonisation of Denominator Data for Primary Health Care Research in Countries of the European Community. IOS Press.

9. Snacken, R., Bensadon, M., Strauss, A., 1995. The CARE telematic network for the surveillance of influenza in Europe. Meth. Inform. Med. 34, 518-522. 
Uphoff, H., Stalleicken, I., Bartelds, A., Phiesel, B., Kistemann, B.T. Are influenza surveillance data useful for mapping presentations?

Virus Research: 103, 2004, nr. 1-2, p. 35-46

10. Uphoff, H., 1998a. A study of reasons for an increase in acute respiratory tract infections reported by influenza sentinel practices in Germany. J. Epidemiol. Commun. Health 1, 43-45.

11. Uphoff, H., 1998b. der "Praxisindex" als eine Größe für regionale Betrachtungen der Influenza-Aktivität. Infektionsepidemiol. Forschg. 3-4, 50-55.

12. Uphoff, H., 1998c. European influenza surveillance scheme (EISS): Eine vereinfachte Darstellung nationaler Influenza-Surveillance-Daten. Infektionsepidemiol. Forschg. 3-4, 4249.

13. Uhoff, H., Phiesel, Kistemann, 2002. Are influenza surveillance data useful for detailed mapping presentations? DAE Tagung in Garmisch, Tagungsberichte der AG räumliche Statistik Heft5, Mai 2002, pp. 105-112.

14. van den Brandhof, W.E., Bartelds, A.I.M., et al., 2001. Surveillance of acute respiratory infections in general practices-The Netherlands, winters 1998/1999 and 1999/2000. RIVM Report No. 217617003. 